\title{
A new platform for gravitational microfluidic using ferrofluids
}

\section{Authors:}

Blandine Bolteau ${ }^{1,2,3}$, Kevin Dos Santos ${ }^{1}$, Frédéric Gelebart ${ }^{1}$, José Gomes ${ }^{1}$, Jérémie Teisseire ${ }^{3}$, Etienne Barthel ${ }^{2}$, Jérôme Fresnais* ${ }^{1}$

${ }^{1}$ Sorbonne Université, CNRS, Laboratoire de Physico-chimie des Electrolytes et Nanosystèmes Interfaciaux, PHENIX - UMR 8234, F-75252 Paris cedex 05, France.

${ }^{2}$ CNRS/UPMC/ESPCI ParisTech-PSL Research University, UMR 7615, Laboratoire SIMM, 10 Rue Vauquelin, 75231 Paris, France

${ }^{3}$ Surface du Verre et Interfaces, UMR 125 CNRS/Saint-Gobain, 39 Quai Lucien Lefranc, F-93303 Aubervilliers, Cedex, France

* Corresponding author: jerome.fresnais@sorbonne-universite.fr 


\section{Abstract}

Among the large variety of microfluidic platforms, surface devices are a world apart. Electrowetting systems are used to control the displacement of droplets among predetermined pathways. More confidential, superhydrophobic surfaces are more and more described as new elements to guide spherical droplet reactors. As such, they can exhibit confinement properties analog to channel-based microfluidics.

In this article, we describe a new strategy to use superhydrophobic surfaces as permanently-tilted microfluidic platform, on which droplets containing iron oxide nanoparticles are guided with permanent magnets. These droplets are fed with water through a capillary tube until their weight exceeds the magnetic field force. Thus, the volume at which the droplet rolls off the surface is only governed by the initial quantity of magnetic nanoparticles and the tilting angle of the surface. This phenomenon provides a strategy for droplet dilution in a simple and reproducible manner, which is not that easy in micro-channels, and a key advantage of open systems.

As a proof of concept, we used this platform to prepare magnetic filaments by a salting-out process already described in large batches. By reducing salt concentration on the platform, we are able to control the electrostatic attractive interactions between iron oxide nanoparticles coated with poly(acrylic acid) and a positively charged polyelectrolyte (poly(diallyldimethylammonium chloride)). The formation of nanostructured filaments was conducted in two minutes while more than 30 minutes were required by dialysis. Our results also illustrate the power of microfluidic reaction processes since such magnetic filaments could not be obtained through direct batch dilution due to mixing issues. Such microfluidic platforms could be useful for the efficient and simple dilution of systems where reactivity is controlled by concentration.

Keywords: superhydrophobic surfaces, ferrofluids, microfluidic, tilted surface 


\section{Introduction}

In the framework of microfluidic, open-channel systems are less used than closed microchannels, even-though their advantages are numerous. They exhibit the potential of confined liquid systems without the detrimental interferences with the micro-channel walls. In this area, electro-wetting systems are interesting because electric field application allows the guidance of droplets on a predetermined pathway ${ }^{1}$. Platforms are described to use trains of droplets that can merge or separate on-demand. More confidential is the use of superhydrophobic surfaces as microfluidic open-channel systems on their own ${ }^{2,3}$. There are some examples where droplets are guided when elastic surfaces are deformed ${ }^{4}$. Fewer examples are given for "ferrofluidics " 5 . The advantages of this technique is that no specific development is required on the hydrophobic corrugated surface itself, and the driving force is low because of the reduced hysteresis of the superhydrophobic state ${ }^{6}$. These ferrofluid droplets can be displaced and deformed by controlling the magnetic field amplitude and gradient ${ }^{7}$. Additionally, recent articles present the use of superhydrophobic magnetic needle to handle magnetic droplets ${ }^{8}$.

Here we propose a new route to use superhydrophobic surfaces as platforms for of milli/microfluidic using ferrofluids not only as mobile droplets but also as reactive ones. Ferrofluid droplets can be moved on surfaces using permanent magnets. These droplets can be fed with solutions containing reactive entities (either molecules, ligands, polymers, proteins, or even cells) that can react (or not) with the magnetic nanoparticles. The dilution that occurs during the droplet feeding is well controlled and is a valuable manner to progressively reach the targeted concentration inside the droplets, which is not easily achieved in closed channels for large dilution rates, except on electro-wetting droplet platforms.

Moreover, a very simple way to obtain reproducible mixing ratios between the on-growing droplet and the reactive feeding is to tilt the surface so that the increasing weight of the droplet eventually reaches a threshold where it overcomes the magnetic confinement. Evidences have been demonstrated in the literature where tilting the surface can move the droplet from one hydrophilic post to another one ${ }^{9}$. The balance between both forces (weight and magnetic coupling) is only driven by the ferrofluid concentration, the starting droplet volume, and the tilting angle of the surface. Thus, reproducible dilution / mixing ratios can be achieved and potentially parallelized using networks of magnets.

\section{Methods}

Superhydrophobic surfaces

Superhydrophobic surfaces were prepared by a soft lithography process using a SU-8 
photosensitive resin mold. It consists of a square network of cylindrical pillars with a diameter of $17.1 \mu \mathrm{m}, 61.6 \mu \mathrm{m}$ high and a spacing of $50 \mu \mathrm{m}$ between the center of pillars. The SU-8 resin was deposited through spin coating (30 s at $500 \mathrm{rpm}$, followed by $30 \mathrm{~s}$ at $1900 \mathrm{rpm}$ ) and preheated at $65^{\circ} \mathrm{C}$ during 3 minutes and $95^{\circ} \mathrm{C}$ during 9 minutes. Exposure through a transparent mask was carried out with 8 cycles of 30 seconds on and 20 seconds off at $70 \%$ of the maximum power of the lamp at $365 \mathrm{~nm}$ in a UV-KUBE. The resin was post-baked at $65^{\circ} \mathrm{C}$ during 2 minutes followed by one 7 minutes at $95^{\circ} \mathrm{C}$ to ensure a complete polymerization of the resin. Development is conducted with SU8-développeur (Propylene Glycol Monomethyl Ether Acetate, PG-MEA). At last, a hard-bake is conducted at $150^{\circ} \mathrm{C}$ during 15 minutes.

Then, SYLGARD 184 was used to prepare a negative mold of the pillars. After activation with an $\mathrm{O}_{2}$ plasma treatment and a silanization with trichloro $(1 \mathrm{H}, 1 \mathrm{H}, 2 \mathrm{H}, 2 \mathrm{H}$ perfluorooctyl)silane in the vapor phase, the mold was used to reproduce the pillar network using SYLGARD 184. It can be re-used several times for further replication of the structures. The surfaces were characterized by Scanning Electron Microscopy (SEM) and optical microscopy (OLYMPUS...). Contact angles (advancing and receding), as well as sliding angles were measured with a portable goniometer (Krüss DSA 100) and a home-made tilting goniometer.

Distilled water was used with droplet volumes ranging from $5 \mu \mathrm{L}$ to $20 \mu \mathrm{L}$.

ImageJ software with Snake plugin was used to measure the contact angles of droplets deposited on the surfaces.

Ferrofluid

The chemicals were used as received: Nitric acid ( $\mathrm{HNO}_{3}, 69.5 \%$ water solution, Carlo Erba), hydrochloric acid $\left(\mathrm{HCl}, 37 \%\right.$ water solution, AnalaR Normapur), iron (II) chloride ( $\mathrm{FeCl}_{2}$, ${ }_{4} \mathrm{H}_{2} \mathrm{O}$, AnalaR Normapur, VWR), iron (III) chloride ( $\mathrm{FeCl}_{3}, 6 \mathrm{H}_{2} \mathrm{O}$, Prolabo), iron (III) nitrate ( $\mathrm{Fe}\left(\mathrm{NO}_{3}\right) 3,9 \mathrm{H}_{2} \mathrm{O}$, Technical, VWR), ammonia aqueous solution $\left(\mathrm{NH}_{4} \mathrm{OH}, 28-30 \%\right.$, SigmaAldrich). Water was high purity grade, obtained by an Elga purification system $(18 \mathrm{M} \Omega \mathrm{cm})$. Aqueous solutions of maghemite MNP ( $\left.\mathrm{Y}-\mathrm{Fe}_{2} \mathrm{O}_{3}\right)$ were synthesized following Massart's coprecipitation method ${ }^{10}$. Ammonia was added to an equimolar acidic solution of iron (II) and iron (III) to induce the co-precipitation into $\mathrm{Fe}_{3} \mathrm{O}_{4}$ (magnetite). It was then boiled for 30 minutes in the presence of iron nitrate in order to oxidize the magnetite into maghemite $(\gamma$ $\mathrm{Fe}_{2} \mathrm{O}_{3}$ ). The resulting dispersion was then washed with acetone and ether before redispersion in an aqueous nitric acid solution.

Iron oxide nanoparticles were coated with poly(acrylic acid) (PAA) using a well-established process $^{11,12}$. In brief, a dispersion of iron oxide at $0.5 \% w t$. (100 $\mathrm{mL}$ ) is added to an acidic 
solution of PAA at $1 \% \mathrm{wt}$. (300 $\mathrm{mL}$ ) and stirred 20 minutes with the use of a magnetic bar. The particles spontaneously aggregate due to their interactions with uncharged PAA. These aggregates are magnetically separated and the supernatant is removed. The $\mathrm{pH}$ of the remaining preparation is raised to 10 . At this $\mathrm{pH}$ value, the nanoparticles are well dispersed and stable in a $\mathrm{pH}$ range of 10 to 5 .

These nanoparticles can be aggregated through their interactions with positive polyelectrolytes. Poly(dialyldimethylammonium chloride) (PDADMAC) was used in this study ${ }^{13}$. To control the attractive electrostatic interactions between nanoparticles and positive polyelectrolyte, brine solution of PDADMAC in $0.5 \mathrm{M} \mathrm{NH}_{4} \mathrm{Cl}$ and a dispersion of PAA-coated nanoparticles at the same ionic strength were prepared. Mixing the two salty solutions, a stable dispersion is obtained for which hydrodynamic size reflects well dispersed PAA-coated nanoparticles. Decreasing the ionic strength through dilution down to $0.1 \mathrm{M}$, aggregates form ${ }^{14}$. If this decrease in conducted in the presence of a magnetic field, the aggregates are elongated ${ }^{15}$.

Ferrofluid droplet guidance

To move ferrofluid droplets on a tilted superhydrophobic surface, magnets was attached onto an oscillating motor that can move with a rotation angle of $180^{\circ}$ max and adjustable tangential velocity ranging from $0.01 \mathrm{~cm} / \mathrm{s}$ up to $4 \mathrm{~cm} / \mathrm{s}$. The motor is driven by an Arduino type set-up and programmed with the Arduino IDE software.

The magnets consist of a column of 2 cylindrical magnets with $1 \mathrm{~cm}$ in diameter and $2 \mathrm{~cm}$ in height and a conical magnet on top (large diameter is $1 \mathrm{~cm}$, small diameter is $0.5 \mathrm{~cm}$ ) made of Neodyn-Bore magnet (SUPERMAGNETE). The distance between magnet and surface (i.e. bottom of the droplet) varies between 2 and $20 \mathrm{~mm}$. 
Results \& Discussion

Measurements with water droplets

At As a first step, PDMS superhydrophobic surfaces (image and structure characterization are given in figure 1) were synthesized according to classical soft photolithography processes $^{16}$. These surfaces are made of arrays of pillars ca. $17 \mu \mathrm{m}$ in diameter and $63 \mu \mathrm{m}$ high. They were characterized in terms of contact angle, contact angle hysteresis, and sliding angle using water. The advancing contact angle was measured at $165^{\circ}$, the receding contact angle was measured at $145^{\circ}$ by tilting the surface until the droplet moves. These two contact angles give an hysteresis of $20^{\circ}$.

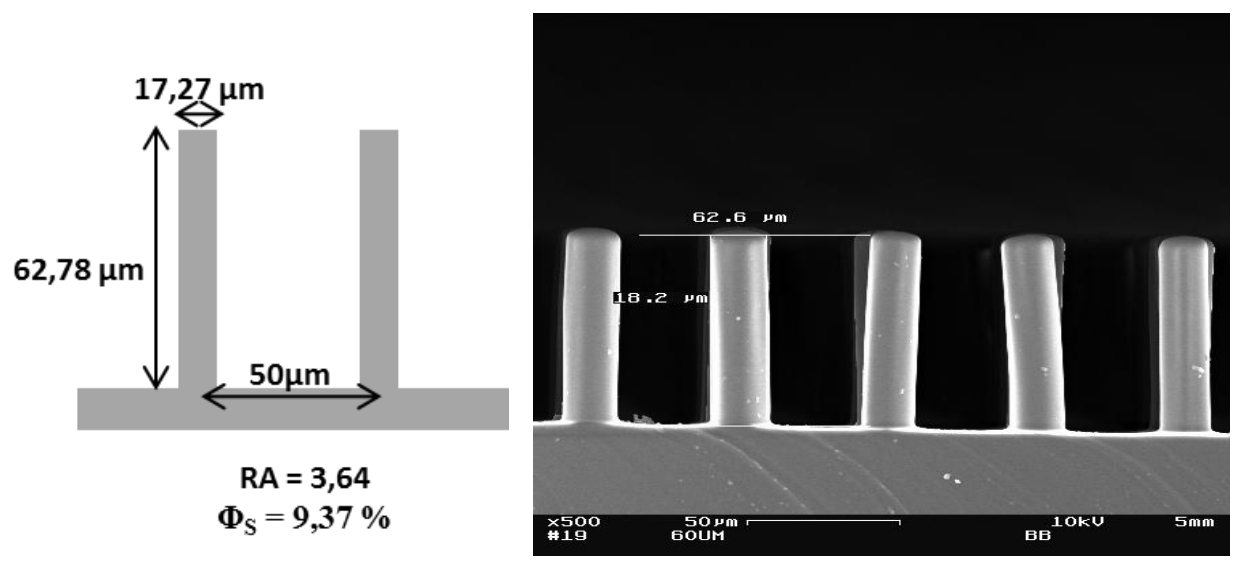

Figure 1: Schematics with pillar geometry; SEM image.

The sliding angles were measured with water droplets from $5 \mu \mathrm{L}$ to $20 \mu \mathrm{L}$. As expected, the smaller the droplets, the higher the sliding angle of the surface (figure 2). These values are in good agreement with the results from the literature on those such model surfaces ${ }^{17,18}$. Indeed, the sliding angles can be fitted comparing the hysteresis value and the weight of the droplet as

$$
m g \sin \alpha>R Y\left(\cos \theta_{r}-\cos \theta_{a}\right),
$$

where $\mathrm{m}$ is the weight of the droplet, $\mathrm{g}$ is the gravitational acceleration, $\mathrm{R}$ is the diameter of the droplet, $Y$ is the surface tension of the liquid and $\theta_{r}$ and $\theta_{a}$ are the receding and advancing contact angles. We found no significant difference for the sliding angles with the ferrofluid droplets which is reasonable as surface tension changes are minute between the different dispersions and water. These measurements are important to evaluate the role of viscous friction for droplet motion. A simple calculation based on Eq. 1, for a $5 \mu \mathrm{L}$ droplet, gives a friction force of the order of $10^{-2} \mu \mathrm{N}$, which is negligible compared to the magnetic force applied to the ferrofluid droplet by the magnet (see calculation below). 


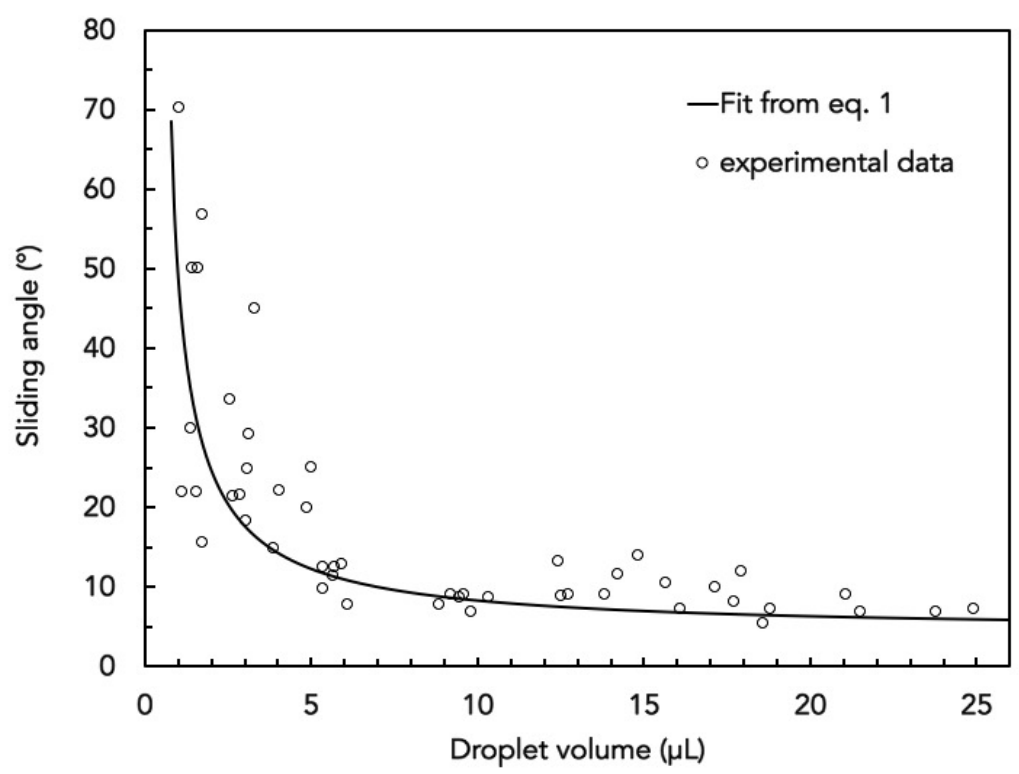

Figure 2: Sliding angle versus droplet volume; line corresponds to equation 1.

Measurement with ferrofluid droplets

Without magnetic forces applied to the droplet, Eq. 1 makes it straightforward to evaluate the tilting angle at which a droplet will roll off. On the contrary, when a magnet is used to confine a ferrofluid droplet, the tilting angle at which the magnetic droplet can be manipulated is larger than the roll-off angle without magnet. Typically, tilting angles of $10^{\circ}$, $20^{\circ}, 40^{\circ}$, and $60^{\circ}$ will be used with ferrofluid droplet volumes ranging from 5 to $20 \mu \mathrm{L}$.

To evaluate the ideal distance between the magnet and the surface to move ferrofluid droplets, we investigated the form of the droplet (figure 3) versus the distance between the top of the magnet and the surface. The deformation of the droplet has already been established on ferrofluid droplets with magnetic fields comparable to ours. Indeed, the maximum magnetic field applied in a previous study ${ }^{7}$ is $370 \mathrm{mT}$, which is in good agreement with our measurements which give a magnetic field amplitude of $335 \mathrm{mT}$ at $2 \mathrm{~mm}$ from the magnet. For such a magnetic field and magnetic field gradient amplitudes, the magnetic force applied to the ferrofluid droplet is:

$$
F=m \frac{\Delta \chi}{\rho \mu_{0}} B \operatorname{gradB}
$$

where $\Delta \chi$ corresponds to the difference of magnetic susceptibility of the ferrofluid, $\mathrm{m}$ is the mass of magnetic materials, $\rho$ is the density of the fluid, $\mu_{0}$ is the permeability constant, $B$ and gradB are the magnetic field and magnetic gradient amplitude respectively. In our case, this magnetic force is of the order of $1 \mu \mathrm{N}$, which is 100 times larger than the typical 
droplet friction forces.
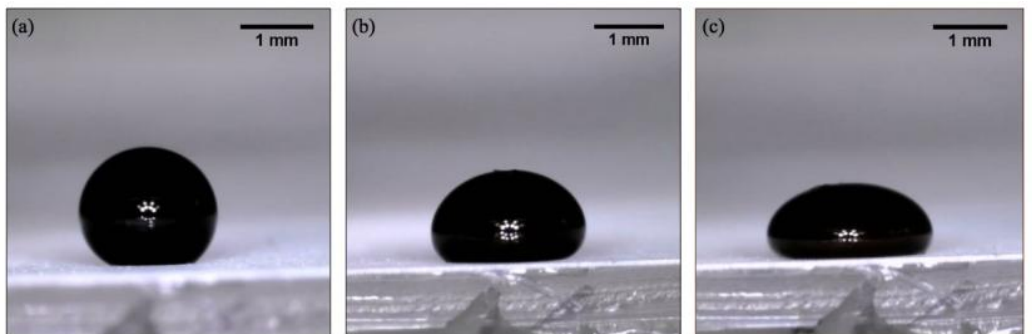

Figure 3: Images of a ferrofluid droplet (0.8 M) at three decreasing magnet / surface distances ( $20 \mathrm{~mm}, 10 \mathrm{~mm}$, and $2 \mathrm{~mm}$ ).

When the magnet approaches the droplet, the droplet flattens as observed by Zhu et al ${ }^{7}$. Additionally, we also measure the contact angle as the magnet gets closer to the ferrofluid droplet. Indeed, for the droplet to be driven by the magnet, the transition to the Wenzel state must be avoided. Figure 4 represents the contact angle of ferrofluid droplets with different concentrations ranging from $0.4 \% \mathrm{wt}$. to $6.4 \% \mathrm{wt}$. We find that the contact angle starts around the mean contact angle $\left(\right.$ ca. $\left.145^{\circ}\right)$ for large magnet-droplet distances. As the magnet approaches and the droplet flattens, the contact angle increases towards the advancing contact angle (ca. $\left.165^{\circ}-170^{\circ}\right)$. In figure $4 \mathrm{a}$, we have reported the variation of the relative aspect ratio of the droplet versus the distance from the magnet for various concentrations. The data are normalized by the aspect ratio of the droplet when the magnet is $20 \mathrm{~mm}$ away. The deformation is larger and appears at a larger distance as the concentration increases. As a consequence, the contact angle varies as the droplet is deformed, following the same dependence with ferrofluid concentration and surface to magnet distance. In contrast to literature reports, no transition toward the Wenzel state is observed in our case, even when the magnet is as close as $2 \mathrm{~mm}$ from the droplet. The good stability of the Cassie Baxter regime is not only due to the low surface fraction ( $9.4 \%$ ) but also to the length of the pillars. Indeed, Al-Azawi et al. demonstrated the transition towards Wenzel state for surface fraction values 5 times larger and for pillars which are $40 \mu \mathrm{m}$ high compared to $63 \mu \mathrm{m}$ in the present study ${ }^{19}$. They reported that the transition towards the Wenzel state was enhanced by oscillations of the droplet on the surface, which were not observed in our experiments. 

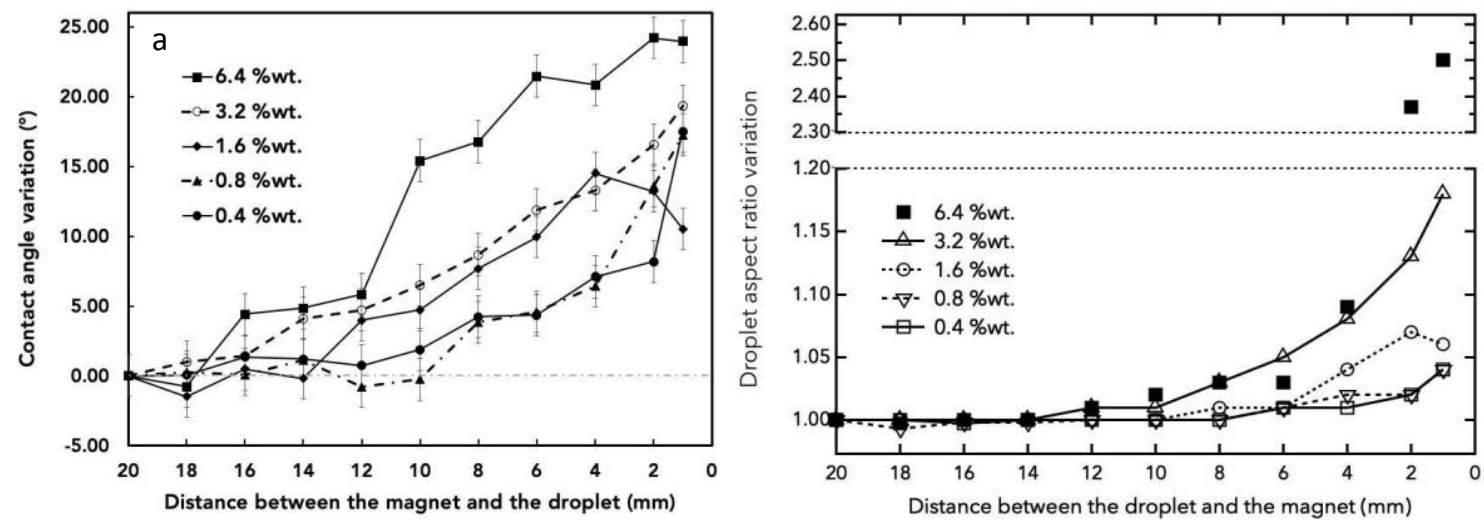

Figure 4: (a) contact angle variation versus distance between the magnet and the droplet for different ferrofluid concentration (from $6.4 \% w t$ t. to $0.4 \% w t$.); (b) relative aspect ratio of the droplet versus the distance between the magnet and the droplet

In order to apply this system to a microfluidic platform, we evaluated the distance at which droplets can be manipulated with the magnet as a function of nanoparticle concentration (figure 5). The distance at which droplets follow the motion of the magnet depends linearly on the starting volume and the tilting angle for droplet concentration larger than $0.8 \% w t$. Below this concentration, it is not possible to move the droplets, whatever the distance between the magnet and the droplet base. We thus chose to work with a ferrofluid concentration of $1 \% \mathrm{wt}$. and a distance between the magnet and the droplet of $4 \mathrm{~mm}$, to ensure active and responsive droplets when the magnet is moved below the surface.

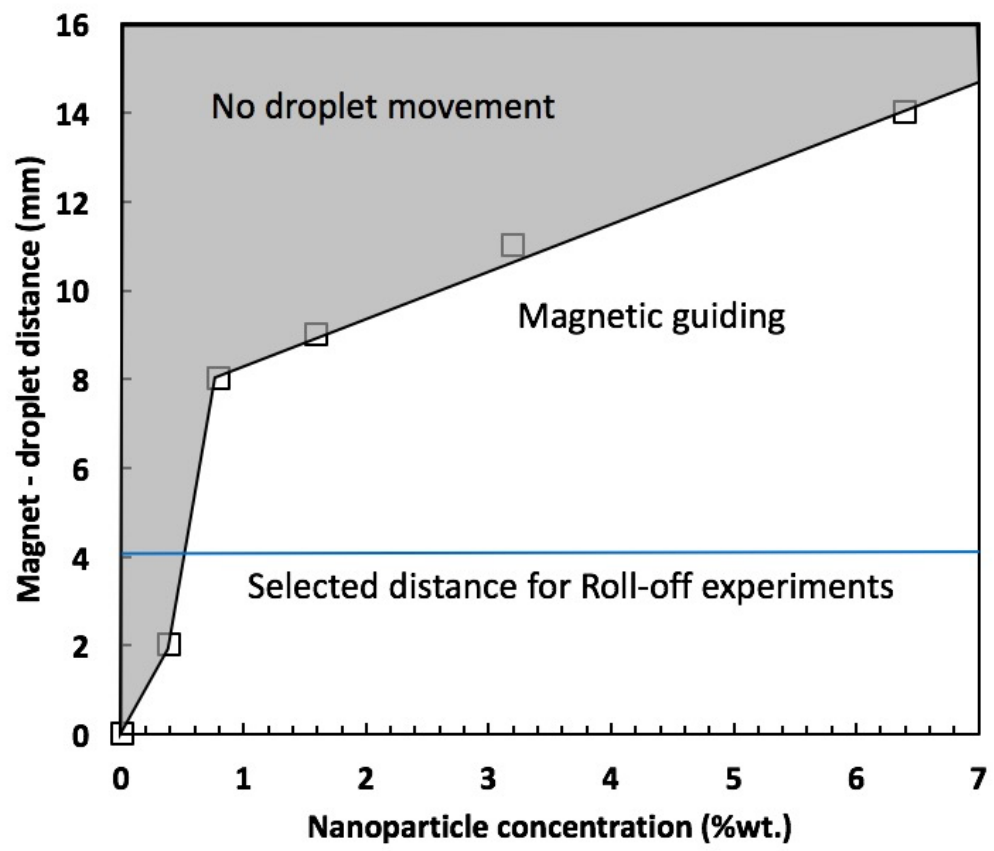

Figure 5: minimal distance between the magnet and the droplet versus nanoparticle concentration (\%wt.) to move the droplet on the surface. The grey area corresponds to the 
distance where no guidance is possible. The blue line corresponds to the distance selected for roll-off experiments.

We investigated the roll-off volume of a ferrofluid droplet moving periodically on a tilted surface. At each period, the droplet was fed with water through a capillary tube situated at the top of the droplet trajectory until the growing droplet escaped from the magnetic confinement and rolled off the surface. Results are reported in figure 6a (error bars correspond to the standard deviation of at least 3 measurements). An important observation is that the volume at roll-off normalized by the initial volume of the droplet is nearly constant. Thus, dilution at roll-off is independent from initial volume. Figure $6 b$ reports dilution ratio versus the tilting angle for a ferrofluid with initial concentration of 1 $\% w t .$, extracted from figure 6a. Therefore, for a given initial ferrofluid concentration, dilution at roll-off strongly depends on tilting angle. With such a device, it is straightforward to select the dilution at droplet roll-off.
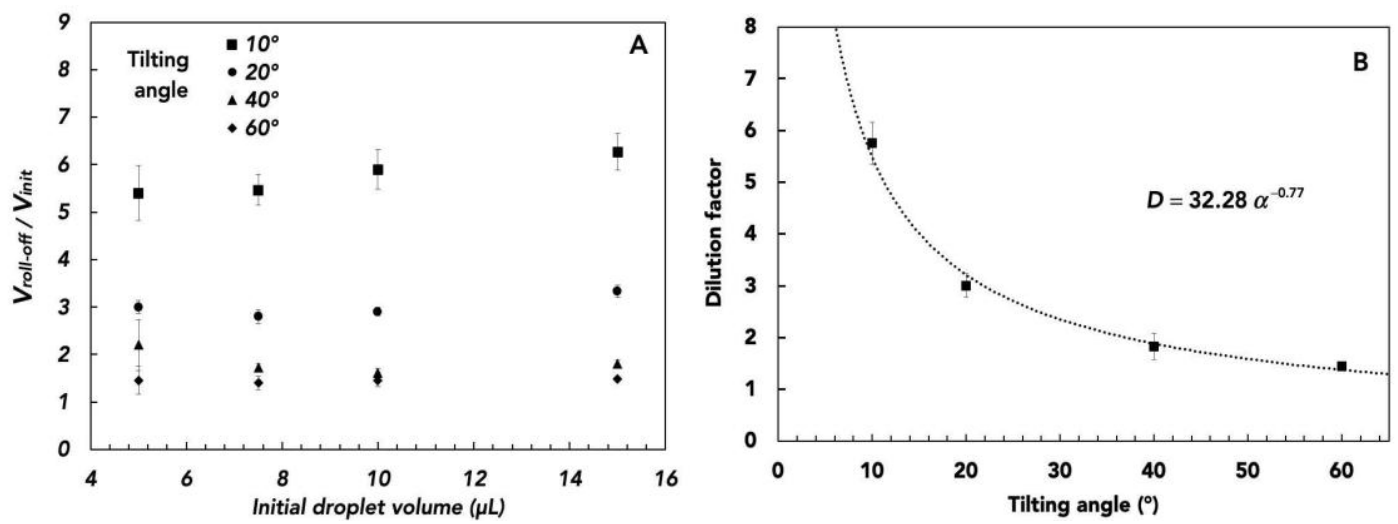

Figure 6: (a) Ratio between roll-off volume and initial volume versus the initial droplet volume at different tilting angles $\left(10^{\circ}, 20^{\circ}, 40^{\circ}\right.$, and $\left.60^{\circ}\right)$ for a ferrofluid at $1 \%$ wt.; (b) dilution factor versus tilting angle

To evaluate the potential of this method for rapid and robust droplet dilution, we investigated the volume at roll-off for a tilting angle of $40^{\circ}$ and different droplet velocities ranging from $8 \mathrm{~mm} / \mathrm{s}$ up to $37.5 \mathrm{~mm} / \mathrm{s}$ (figure 7). The roll-off volumes are found to be superimposed for the different initial ferrofluid droplets, whatever the droplet velocity. These velocities can be compared to droplet generation and flows in micro-channel in biphasic systems where flow rates as high as $150 \mathrm{~mm} / \mathrm{s}$ are measured. Thus, manipulation of droplets with our device can be considered for the dilution of numerous droplets in short times. 


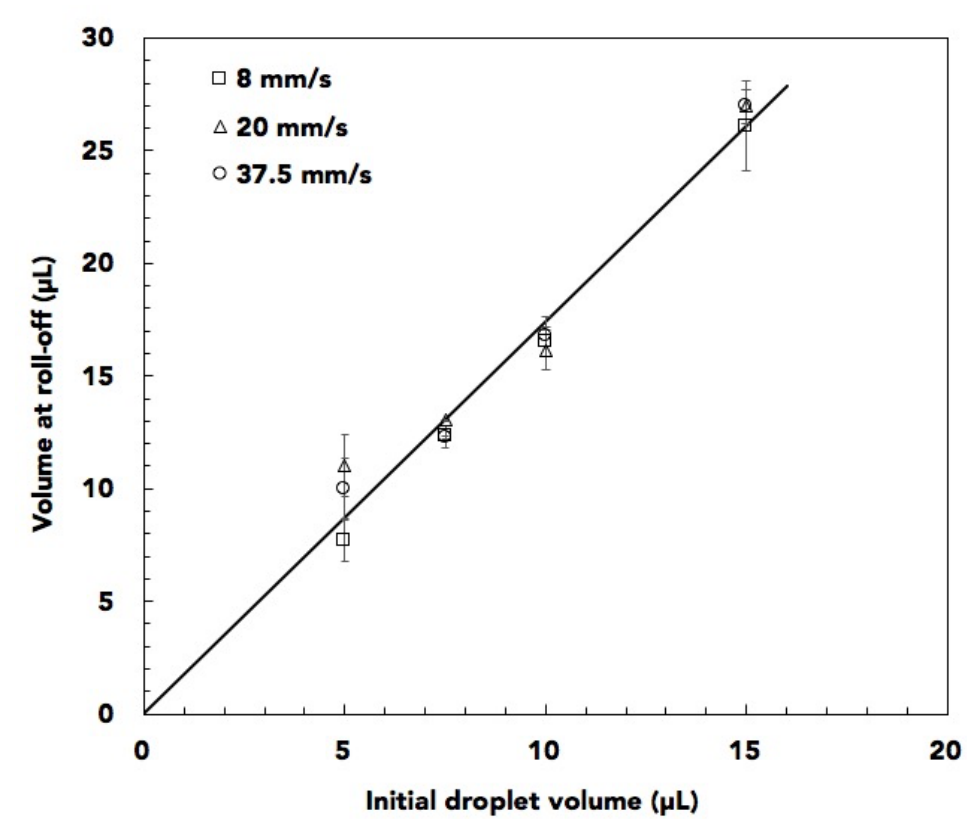

Figure 7: Total volume at roll-off versus initial droplet volume at different droplet velocities $(8,20$, and $37.5 \mathrm{~mm} / \mathrm{s})$ for a tilting angle of $40^{\circ}$ (at the concentration of $1 \% w t$.). The line corresponds to a linear fit.

Measurements with reactive magnetic droplets

As a proof of concept, we investigated the reaction of iron oxide nanoparticles towards electrostatic complexation with oppositely charged polyelectrolytes. Indeed, we have previously demonstrated a controlled pathway to tune the interaction between positively charged polyelectrolytes and negatively charged nanoparticles ${ }^{14}$. Addition of a large quantity of salt (ca. $\mathrm{NH}_{4} \mathrm{Cl}$ at $0.5 \mathrm{M}$ ) in a dispersion of poly(acrylic acid) coated nanoparticles and in a positively-charged polyelectrolyte solution screens their interaction when they are mixed. Dilution with water decreases salt concentration below a threshold at which attractive electrostatic interactions are revealed. We have previously demonstrated that rods can be obtained in 1 hour through the dialysis of the salted mixture in the presence of a magnetic field ${ }^{13}$.

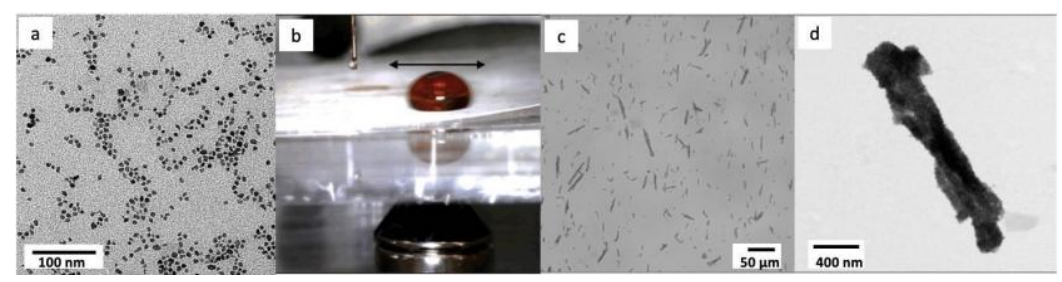

Figure 8: Image of a droplet containing a salted ferrofluid with positively charged polyelectrolytes (b). The capillary tube delivers water so that interparticle electrostatic interactions are revealed by salt dilution. Optical microscopy (c) and TEM images of the 
pristine nanoparticles (a) and as-synthesized rods (d).

Here we investigated the dilution process in a droplet of given initial volume guided by the magnet and diluted with water through a capillary tube. Figure $8 \mathrm{~b}$ represents the picture of the droplet containing the magnetic nanoparticles as well as the positive polyelectrolyte and salt. Dilution occurs at every passage of the droplet above the capillary tube. Interestingly, the magnetic nanoparticles are not uniformly distributed in the droplet while water is added to the moving droplet. This is due to the strong magnetic interaction between the nanoparticles and the magnet. On the contrary, the non-magnetic species (salts, polyelectrolytes) can diffuse freely inside the droplet, which allows the formation of the aggregates at the interface where the salt concentration reaches the critical value $(0.5$ $\mathrm{M}$ of $\mathrm{NH}_{4} \mathrm{Cl}$ ). This phenomenon will be studied in a forthcoming work.

At the end of the dilution (the volume of the droplet is increased 10 times from its initial volume), the droplets are collected and their content is analyzed by optical (figure 8-c) and transmission electron microscopy (figure 8-d). As a comparison, image 8a represents the pristine nanoparticles to be co-assembled. We evidenced the synthesis of elongated aggregates as found in our previous work ${ }^{13}$. In previous attempts, these aggregates could not be obtained through a dilution process, due to the hydrodynamic perturbations that usually occur when two liquids are mixed together and also because of the rapid assembly rate between oppositely charged species above the critical salt concentration. This is thus the first evidence of the synthesis of those elongated aggregates through a dilution process. Moreover, assembly is obtained within minutes instead of a one-hour dialysis.

Table 1 reports the mean lengths and their corresponding standard deviations for different starting droplet volumes from 5 to $30 \mu \mathrm{L}$. The bigger the droplet, the longer the rods. The differences observed in length are not that large but it could be possible to change drastically their length by changing the strength of the magnetic field (the distance between the droplet and the magnet) or the concentration of ferrofluid.

Table 1: magnetic rod mean lengths and standard deviation of rods versus initial droplet volume

\begin{tabular}{|c|c|c|}
\hline Initial droplet volume $(\mu \mathrm{L})$ & Rods mean length $(\mu \mathrm{m})$ & Standard deviation \\
\hline 5 & 9.8 & 3.8 \\
\hline 10 & 11.8 & 3.6 \\
\hline 20 & 11 & 5.3 \\
\hline 30 & 13.2 & 6.3 \\
\hline
\end{tabular}




\section{Conclusion}

To conclude, we propose a simple and robust method to perform dilution in microfluidic systems using ferrofluid droplets moving on a tilted superhydrophobic surface. Control over progressive dilution is not easy to achieve within microfluidic systems. Here, dilution at rolloff only depends on the tilting angle and not on the initial droplet volume, and is very reproducible for various velocities up to $37.5 \mathrm{~mm} / \mathrm{s}$. This dilution process was successfully used to prepare nanostructured rods of ca. $10 \mu \mathrm{m}$ long in a few minutes. Similar rods cannot be obtained by direct dilution of the bulk solution and their synthesis takes one hour through dialysis.

This dilution set-up could be useful to achieve dilution of solutions where a solute can react with the surface of iron oxide nanoparticles. For example, investigation of kinetic adsorption of molecules of interest such as proteins or cancer markers in blood drops could be achieved rapidly and in a reproducible manner. 


\section{Acknowledgements}

[Insert text here] 


\section{References}

(1) Pollack, M. G.; Shenderov, A. D.; Fair, R. B. Electrowetting-Based Actuation of Droplets for Integrated Microfluidics. Lab. Chip 2002, 2 (2), 96-101.

https://doi.org/10.1039/b110474h.

(2) Seo, K. S.; Wi, R.; Im, S. G.; Kim, D. H. A Superhydrophobic Magnetic Elastomer Actuator for Droplet Motion Control. Polym. Adv. Technol. 2013, 24 (12), 1075-1080. https://doi.org/10.1002/pat.3190.

(3) Oliveira, N. M.; Neto, A. I.; Song, W.; Mano, J. F. Two-Dimensional Open Microfluidic Devices by Tuning the Wettability on Patterned Superhydrophobic Polymeric Surface. Appl. Phys. Express 2010, 3 (8), 85205.

(4) Nagappan, S.; Ha, C.-S. Emerging Trends in Superhydrophobic Surface Based Magnetic Materials: Fabrications and Their Potential Applications. J Mater Chem A 2015, 3 (7), 3224-3251. https://doi.org/10.1039/C4TA05078A.

(5) Bormashenko, E.; Pogreb, R.; Bormashenko, Y.; Musin, A.; Stein, T. New Investigations on Ferrofluidics: Ferrofluidic Marbles and Magnetic-Field-Driven Drops on Superhydrophobic Surfaces. Langmuir 2008, 24 (21), 12119-12122. https://doi.org/10.1021/la802355y.

(6) Cassie, A. B. D.; Baxter, S. Wettability of Porous Surfaces. Trans. Faraday Soc. 1944, 40, 546-551.

(7) Zhu, G.-P.; Nguyen, N.-T.; Ramanujan, R. V.; Huang, X.-Y. Nonlinear Deformation of a Ferrofluid Droplet in a Uniform Magnetic Field. Langmuir 2011, 27 (24), 14834-14841. https://doi.org/10.1021/la203931q.

(8) Yang, C.; Ning, Y.; Ku, X.; Zhuang, G.; Li, G. Automatic Magnetic Manipulation of Droplets on an Open Surface Using a Superhydrophobic Electromagnet Needle. Sens. Actuators B Chem. 2018, 257, 409-418. https://doi.org/10.1016/j.snb.2017.10.118.

(9) Kong, T.; Brien, R.; Njus, Z.; Kalwa, U.; Pandey, S. Motorized Actuation System to Perform Droplet Operations on Printed Plastic Sheets. Lab. Chip 2016, 16 (10), 1861-1872. https://doi.org/10.1039/C6LC00176A.

(10) Bee, A.; Massart, R.; Neveu, S. Synthesis of Very Fine Maghemite Particles. J Magn Magn Mat 1995, 149 (1-2), 6-9.

(11) Sehgal, A.; Lalatonne, Y.; Berret, J.-F.; Morvan, M.

Precipitation-Redispersion of Cerium Oxide Nanoparticles with Poly(Acrylic Acid):

Toward Stable Dispersions. Langmuir 2005, 21 (20), 9359-9364. https://doi.org/10.1021/la0513757.

(12) Guibert, C.; Dupuis, V.; Fresnais, J.; Peyre, V. Controlling Nanoparticles Dispersion in Ionic Liquids by Tuning the PH. J. Colloid Interface Sci. 2015, 454 (0), 105-111. https://doi.org/10.1016/j.jcis.2015.04.059.

(13) Yan, M.; Fresnais, J.; Sekar, S.; Chapel, J.-P.; Berret, J.-F. Magnetic Nanowires Generated via the Waterborne Desalting Transition Pathway. ACS Appl. Mater. Interfaces 2011, 3 (4), 1049-1054. https://doi.org/10.1021/am101188y.

(14) Fresnais, J.; Lavelle, C.; Berret, J. F. Nanoparticle Aggregation Controlled by Desalting Kinetics. J Phys Chem C 2009, 113 (37), 16371-16379.

(15) Fresnais, J.; Berret, J. F.; Frka- Petesic, B.; Sandre, O.; Perzynski, R. Electrostatic Co- Assembly of Iron Oxide Nanoparticles and Polymers: Towards the Generation of Highly Persistent Superparamagnetic Nanorods. Adv. Mater. 2008, 20 (20), 3877-3881.

(16) du Roure, O.; Dequidt, C.; Richert, A.; Austin, R. H.; Buguin, A.; Chavrier, P.; 
Silberzan, P.; Ladoux, B. Microfabricated Arrays of Elastomeric Posts to Study Cellular Mechanics. In Conference on Microfluidics, BioMEMS and Medical Microsystems II; Woias, P. P. I., Ed.; San Jose, CA, 2004; pp 26-34. https://doi.org/10.1117/12.530688.

(17) Joanny, J. F.; de Gennes, P. G. A Model for Contact Angle Hysteresis. J. Chem. Phys. 1984, 81 (1), 552. https://doi.org/10.1063/1.447337.

(18) A L Dubov and K Perez-Toralla and A Letailleur and E Barthel and J Teisseire. Superhydrophobic Silica Surfaces: Fabrication and Stability. J. Micromechanics Microengineering 2013, 23 (12), 125013.

(19) Al-Azawi, A.; Latikka, M.; Jokinen, V.; Franssila, S.; Ras, R. H. A. Friction and Wetting Transitions of Magnetic Droplets on Micropillared Superhydrophobic Surfaces. Small 2017, 13 (38), 1700860. https://doi.org/10.1002/smll.201700860.
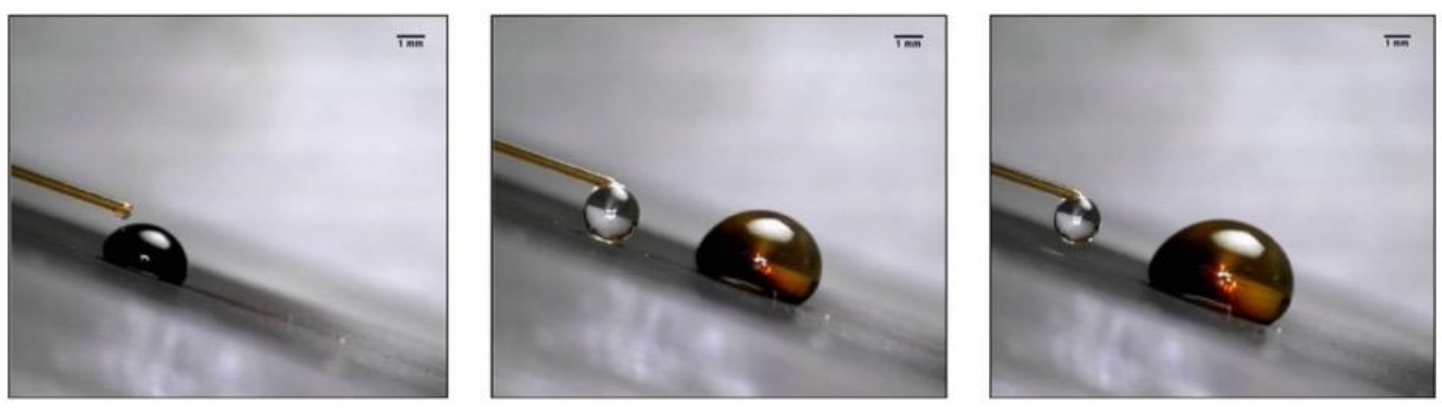